\title{
Harmonic Currents Excited by an Electromagnetic Wave in a Plasma
}

\author{
Lewis Wetzel ${ }^{1}$ and Ting-Wei Tang ${ }^{2}$
}

\author{
Brown University, Providence 12, R.I.
}

(Received October 28, 1964)

\begin{abstract}
This paper reviews the major sources of harmonic currents excited by an electromagnetic wave in an iostropic plasma. The discussion is based on the assumption that a plane wave of prescribed form interacts with a plasma whose response is described by the usual macroscopic equations for the electron velocity, density and temperature. Nonlinear interactions arising from (i) the magnetic field of the plane wave, (ii) the dependence of the collision frequency and other rate parameters on the electron temperature, and (iii) the oscillating space-charge induced by the wave in regions of inhomogeneity, are examined in sufficient detail to provide an estimate of their relative importance in producing harmonic currents in various situations. The physical origins of these currents are discussed and a characteristic perturbation parameter provided for each of the interactions.
\end{abstract}

\section{Introduction}

When a high-frequency electromagnetic wave interacts with a plasma, there are various weak nonlinearities through which harmonic currents can be excited, and these currents may, in turn, give rise to secondary radiation fields. Although certain related nonlinear interactions have been studied in detail, mostly in connection with wave interaction effects in the ionosphere 「Ginsburg and Gurevich, 1960], the literature on harmonic excitation itself is relatively sparse and widely scattered. The reason for this is fairly obvious: the nonlinear sources responsible for the appearance of harmonic currents are weakly excited and are therefore of little interest under most circumstances. Put another way, the linearized theory of wave propagation in a plasma works extremely well. However, just as the availability of intense laser fields at optical frequencies has led to an interest in the nonlinear optics of crystalline media [Armstrong et al., 1962; Bloembergen, 1963], so also should the increasing power of radio and radar transmitters and the long path lengths involved in space communications lead to increased interest in the relatively weak excitation of harmonic signals in ionized media.

Up to the present time the three main practical motives for studying the excitation of such signals have been: (1) the production of spurious signals in the ionosphere [Feinstein, 1950; Försterling and Wüster, 1951; Vilenskii, 1953; Wetzel, 1963; Rydbeck, 1963]; (2) the design, or explanation, of useful harmonic

\footnotetext{
* This work was supported by the Air Force Cambridge Research Laboratories, Office of Aerospace Research, under Contract No. AF $-19(628)-2498$.

${ }^{1}$ Present address: Institute for Defense Analyses, Arlington, Va.

${ }^{2}$ Present address: Department of Aerospace Engineering, University of Connecticut. Storrs, Conn.
}

generators [Maiman, 1957; Uenohara et al., 1957; Baird and Coleman, 1961; Swan, 1961]; (3) the pos. sibility of diagnostic applications [Wetzel, 196la; Rydbeck, 1963]. Harmonic spectra have been observed experimentally in a variety of circumstances, but their origins are seldom clear. For example, in those cases in which a steady magnetic field was present, exciting fields at the gyromagnetic frequency of the electrons have produced observable harmonic signals, but while the cavity measurements made by Maiman [1957] are consistent with a simple and reasonable theory, the more complicated computer calculations of Whitmer and Barrett [1961] are at variance with the experimental results in several respects. Harmonics produced in a microwave dis. charge without a steady magnetic field have been ascribed in one case to strongly inhomogeneous electric fields [Swan, 1961] and in another to modulation of the ionization frequency [Baird and Coleman, 1961]; in the latter case the proposed hypothesis accounts very well for the observed data, but in the form used it appears to lack any convincing theoretical basis. The general picture appears to be that while detectable harmonics can be produced quite easily, the nonlinear processes underlying their production are not usually well understood and most probably occur in combination.

In an effort to bring some order to this picture, we will attempt in this paper to examine a certain class of nonlinearities which might be responsible for some of these harmonic spectra. Because of the complexity of the analysis in the anisotropic case, we will restrict our attention to isotropic plasmas. A steady magnetic field can be expected to enhance certain of the isotropic interactions in the neighborhood of cyclotron resonance, as well as to introduce additional harmonic terms having a different character. When appropriate, we will mention such effects in passing. 


\section{Basic Formulation}

Several authors have examined certain features of the various nonlinear interactions between a plasma and an electromagnetic field on the basis of kinetic theory, using the Boltzmann equation [Margenau and Hartman, 1948; Vilenskii, 1953; Fain, 1955; Rosen, 1961]. This approach has definite virtue when studying a single interaction in detail, but is far too cumbersome if one wishes to evaluate the comparative effects of several nonlinearities within a given mathematical framework. Accordingly, we will choose a simpler, and physically more transparent, description of the plasma in terms of the particle and momentum transport equations, augmented by a phenomenological energy equation. The primary electromagnetic wave will be assumed, when possible, to have the form of a transverse plane wave with prescribed local parameters.

The total current density due to charge motion in the plasma is defined as

$$
\vec{j}=-e\left(\overrightarrow{n_{e} v_{e}}-n_{i} v_{i}\right)
$$

where $-e$ is the electron charge and $n_{e}, \vec{v}_{e}$ and $n_{i}, \vec{v}_{i}$ denote the density and average velocity of the electrons and ions respectively. Since the densities are roughly the same and the ion velocity is smaller than the electron velocity by the factor $m / M$, the mass ratio of electron to ion, we may neglect the ion current and regard the ions as merely providing a uniform neutralizing background. Henceforth, then, we will be interested only in the electron current which, dropping subscripts, will be written

$$
\vec{j}=-\overrightarrow{e n v}
$$

Harmonic components in this current will arise from harmonic components in $n$ and $\vec{v}$, so we must examine the nonlinearities in the equations governing the response of the plasma to the applied electromagnetic field; namely, the equation for the electron velocity

$\frac{\partial v}{\partial t}+\vec{v} \cdot \nabla \vec{v}=-\frac{e}{m}(\vec{E}+\vec{v} \times \vec{B})-\frac{\nabla p_{e}}{m n}-\nu_{c}\left(T_{e}\right) \vec{v}$

the continuity equation

$$
\frac{\partial n}{\partial t}+\nabla \cdot(\overrightarrow{n v})=0
$$

and, to close the system, an equation for the electron energy (written here as a phenomenological equation for the electron temperature [see, e.g., Ginsburg and Gurevich, 1960]):

$$
\frac{\partial T_{e}}{\partial t}=-\frac{2}{3} \frac{e}{k} \vec{v} \cdot \vec{E}-\delta \nu_{c}\left(T_{e}\right)\left[T_{e}-T_{0}\right] .
$$

It has been assumed in the moment equation (2.3) that the electron velocity distribution is isotropic, so the divergence of the stress tensor has been written as the gradient of the isotropic pressure $p_{e}=n k T_{e}$, where $k$ is the Boltzmann constant. In the last term of (2.3) the temperature dependence of the effective collision frequency is noted explicitly. The temperature equation (2.5), if multiplied by $(3 / 2) n k$, simply states that the rate of increase of the average electron energy per unit volume is equal to the difference between the electric power delivered to the electrons and the rate at which energy is lost by collisions with heavy particles held at the equilibrium temperature $T_{0}, \delta$ being the energy loss parameter $(\delta=2 \mathrm{~m} / M$ for elastic collisions, where $M$ is the mass of the heavy particle).

Rather than expand everything in Fourier series and then attempt to disentangle the sources of harmonics from the resulting confusion, we will seek instead to identify the individual sources of harmonics in physical terms and to provide a characteristic measure of the relative importance of each. These sources can be anticipated from an examination of the equations (2.3) to $(2.5)$. The plasma is driven by the prescribed field $\vec{E}$ (and $\vec{B}$ ) and its response appears in the variables $\vec{v}, n$, and $T_{e}$. We know even from simple orbit theory that the magnetic field $\vec{B}$ of the plane wave could perturb the trajectory of the electron in such a way that its motion relative to the phase plane of the wave might be anharmonic. Such a nonlinearity would be "intrinsic" in the sense that it would always be present when a wave propagates in a plasma, and would thus provide a threshold of interest in evaluating the importance of other nonlinearities.

A nonlinearity like the $\vec{v} \cdot \nabla \vec{v}$ term in (2.3) cannot itself be regarded as a primary source of harmonics, although it will affect the detailed response of the plasma to those primary sources which produce changes of electron velocity along the streamlines. Behavior of this latter type might arise from pressure gradients produced by a harmonic temperature wave traveling with the primary wave, or from space-charge variations induced when the primary wave encounters an inhomogeneity. Moreover, the existence of temperature oscillations could introduce harmonic components into $\vec{v}$ through the $T_{e^{-} \text {dependence of the }}$ collision frequency in the last term of (2.3).

Our general approach will be to use the formulation provided by (2.3) to (2.5) to find a semiquantitative description of the effect of these various sources, treating each as if all the others could be ignored. It will be convenient to have a zeroth-order approximation at hand, representing the linearized solution to (2.3) to (2.5) for the case of constant electron temperature, pressure and density. If the primary transverse plane wave is written as

$$
\vec{E}(\vec{r}, t)=\overrightarrow{E_{0}} e^{-\alpha \hat{\kappa} \cdot \vec{r}} \cos (\omega t-\beta \hat{\kappa} \cdot \vec{r})
$$

where $\vec{E}_{0}$ is a constant vector, $\hat{\kappa}$ is the direction of propagation $\left(\vec{E}_{0} \cdot \hat{\kappa}=0\right), \omega$ is the wave frequency and $\beta$ and $\alpha$ are the propagation and attenuation constants 
in a uniform plasma of density $n_{0}$, then the solution of (2.3) in the zeroth-order approximation becomes

$\vec{v}^{(0)}=-\frac{(e / m) \overrightarrow{E_{0}} e^{-\alpha \hat{\kappa} \cdot \vec{r}}}{\left(\omega^{2}+\nu_{c}^{2}\right)}$

$$
\left[\omega \sin (\omega t-\beta \hat{\kappa} \cdot \vec{r})+\nu_{c} \cos (\omega t-\beta \hat{\kappa} \cdot \vec{r})\right]
$$

and from (2.2) the corresponding induced current is $\vec{j}(0)=-e n_{0} \vec{v}^{(0)}$. This zeroth-order current is just the linear polarization current produced by the primary wave in the plasma and thus determines the values of $\beta$ and $\alpha$ for this wave. In addition to generating harmonic currents, the nonlinearities will also perturb the propagation of the primary wave by producing slight changes in $\beta$ and $\alpha$.

\section{Intrinsic Electric-Magnetic Field Interaction}

A free electron in the field of a plane wave experiences both electric and magnetic forces, and although these forces vary with time, it is in the nature of a plane wave that the average of $\vec{E} x \vec{B}$ always points in the direction of propagation $\hat{\kappa}$, so the electron should experience a drift in the $\hat{\kappa}$-direction. Since the trajectory of the electron is now curved in the plane of polarization (established by $\vec{E}$ and $\hat{\kappa}$ ), the projections of its velocity on the phase plane will no longer be sinusoidal - in fact, on the basis of symmetry we would expect that a Fourier representation of the motion would contain odd harmonics only.

These conclusions can be made more precise by solving a reduced version of (2.3) as completely as possible. Let a plane wave whose electric vector is given by (2.6) propagate in the $z$-direction with $\vec{E}_{0}$ $=E_{0} \hat{e}_{x}$, so that $\vec{E}(z, t)=\hat{e}_{x} E_{0} \exp (-\alpha z) \cos (\omega t-\beta z)$. In rationalized MKS units, the associated magnetic field will have the form

$$
\vec{B}(z, t)=\hat{e}_{y} c_{p}^{-1} E_{0} \exp (-\alpha z) \cos (\omega t-\beta z-\phi)
$$

where $c_{p}$ is the wave velocity in the plasma and the phase angle $\phi$ takes account of the phase shift of the magnetic field in a lossy medium and is defined by $\tan \phi=\alpha / \beta$.

An approximation to $\vec{v}$ which accounts only for the $\vec{v} \times \vec{B}$ effect can be found by neglecting $\vec{v} \cdot \nabla \vec{v}$ and $\nabla p_{e}$ in (2.3), assuming that $\nu_{c}$ is a constant, and writing the resulting reduced equation in coordinate form:

$$
\begin{gathered}
\frac{\partial v_{x}}{\partial t}+v_{c} v_{z}=-\frac{e}{m} E(z, t)+\frac{e}{m} v_{z} B(z, t) \\
\frac{\partial v_{z}}{\partial t}+\nu_{c} v_{z}=-\frac{e}{m} v_{x} B(z, t) .
\end{gathered}
$$

Under steady-state conditions there will be no $v_{y}$ component, so we may define a new variable $u=v_{x}+i v_{z}$ and combine (3.1) and (3.2) into a single equation

$$
\frac{\partial \tilde{u}}{\partial t}+\left[\nu_{c}+\frac{i}{c_{p}} \frac{e}{m} E_{0}(z) \cos (\omega t-\beta z-\phi)\right] \tilde{u}=-\frac{e}{m} E(z, t)
$$

for the complex variable $\tilde{u}$. (For ease of writing, we have used the abbreviation $E_{0}(z)$ for $E_{0} \exp (-\alpha z)$.) By the use of an integrating factor, the steady-state (particular) solution of this equation becomes

$$
\begin{gathered}
\tilde{u}=-\exp \left[-\nu_{c} t-i \frac{e E_{0}(z)}{c_{p} m \omega} \sin (\omega t-\beta z-\phi)\right] \\
\times \frac{e}{m} E_{0}(z) \int \cos (\omega t-\beta z) \exp \left[\nu_{c} t-i \frac{e E_{o}(z)}{c_{p} m \omega}\right.
\end{gathered}
$$

$$
\sin (\omega t-\beta z-\phi)] d t
$$

The exponential factors containing $\sin (\omega t-\beta z-\phi)$ can be replaced by the Bessel function expansion

$$
\begin{aligned}
\exp \left[ \pm i \frac{e E_{0}(z)}{c_{p} m \omega} \sin (\omega t-\beta z-\phi)\right] \\
=\sum_{n=-\infty}^{\infty} J_{n}\left(v_{0} / c_{p}\right) \exp [ \pm i n(\omega t-\beta z-\phi)]
\end{aligned}
$$

where $J_{n}$ is the Bessel function of the first kind of order $n$, and we define the parameter $v_{0}=e E_{0}(z) / m \omega$.

Upon performing the integration term by term, multiplying the series together and separating the harmonic terms into real and imaginary parts, the orthogonal velocity components $v_{x}$ and $v_{z}$ may be written in the following approximate form:

$$
\begin{aligned}
v_{x}=- & v_{0}\left\{J_{0}^{2}[\sin (\omega t-\beta z)+\Delta \cos (\omega t-\beta z)]\right. \\
& +\left(\frac{4}{3} J_{0} J_{2}-\frac{1}{2} J_{1}^{2}\right) \sin 3\left(\omega t-\beta z-\frac{2}{3} \phi\right) \\
& +\left(\frac{10}{9} J_{0} J_{2}-\frac{1}{4} J_{1}^{2}\right) \Delta \cos 3\left(\omega t-\beta z-\frac{2}{3} \phi\right) \\
& +\ldots(n \text { odd }) \ldots .\}
\end{aligned}
$$

$$
v_{z}=+v_{0}\left\{J_{0} J_{1}\left[\left(\Delta^{-1}-\Delta\right) \sin \phi+\cos \phi\right]\right.
$$

$$
\begin{array}{r}
+\frac{1}{2} J_{0} J_{1}\left[\cos 2\left(\omega t-\beta z-\frac{1}{2} \phi\right)-\Delta \sin 2\left(\omega t-\beta z-\frac{1}{2} \phi\right)\right] \\
+\ldots(n \text { even }) . . .\} .
\end{array}
$$

Only the lowest order Bessel functions have been retained, and the solution contains only terms through first order in the parameter $\Delta=\nu_{c} / \omega$, which will henceforth be assumed to be small. Note that the transverse velocity $v_{x}$ contains only odd harmonics, while the longitudinal velocity $v_{z}$ contains only even harmonics. It should be emphasized that these are solutions of (3.1) and (3.2), not of (2.3). The existence 
of $v_{z}$ means that both $\vec{v} \cdot \nabla \vec{v}$ and $\nabla p_{e}$ should be included in any attempt to solve (2.3). On the other hand, one expects that a perturbation solution of (2.3) based on the velocities given above would not alter the results in a qualitative way, leading at most to a correction of some of the numerical coefficients.

Since the parameter $v_{0}$ is the magnitude of the unperturbed electron velocity in the case $\nu_{c}=0$, and $c_{p}$ is always greater than the speed of light in vacuum, the ratio $v_{0} / c_{p}$ is small for nonrelativistic velocities and we can use the small-argument approximations for the Bessel functions:

$$
J_{n}\left(v_{0} / c_{p}\right) \simeq \frac{\left(v_{0} / c_{p}\right)^{n}}{2^{n} n !},\left(v_{0} / c_{p}\right)<<1
$$

If these approximate forms are used in (3.6) and (3.7), the expansions can be expressed as power series in the small parameter $\epsilon=\left(v_{0} / c_{p}\right)$ :

$$
\begin{aligned}
v_{x} \simeq-v_{0}[\sin (\omega t-\beta z)+\Delta \cos (\omega t-\beta z)] \\
-\frac{1}{24} \epsilon^{2} v_{0}\left[\sin 3\left(\omega t-\beta z-\frac{2}{3} \phi\right)\right. \\
\left.+\frac{11}{6} \Delta \cos 3\left(\omega t-\beta z-\frac{2}{3} \phi\right)\right]+0\left(\epsilon^{4}\right)
\end{aligned}
$$

$v_{z} \simeq \frac{1}{2} \epsilon v_{0}\left[\cos \phi+\left(\Delta^{-1}-\Delta\right) \sin \phi\right]$

$$
\begin{aligned}
& +\frac{1}{4} \epsilon v_{0}\left[\cos 2\left(\omega t-\beta z-\frac{1}{2} \phi\right)\right. \\
& \left.-\frac{3}{2} \Delta \sin 2\left(\omega t-\beta z-\frac{1}{2} \phi\right)\right]+0\left(\epsilon^{3}\right) .
\end{aligned}
$$

(It should be remarked that $\sin \phi / \Delta$ remains finite for $\Delta=0$.) However, according to (2.4) the appearance of a second-harmonic velocity in the longitudinal direction implies the excitation of a corresponding spacecharge wave:

$$
\frac{\partial n_{2}}{\partial t}=-n_{0} \frac{\partial v_{z 2}}{\partial z}
$$

where numerical subscripts are introduced to identify harmonic components of the various plasma parameters.

Substituting from the second term of (3.10), we obtain

$$
\begin{aligned}
n_{2} \simeq-\frac{1}{4} \epsilon^{2} n_{0}\left[\cos 2\left(\omega t-\beta z-\frac{1}{2} \phi\right)\right. \\
\left.-\frac{3}{2} \Delta \sin 2\left(\omega t-\beta z-\frac{1}{2} \phi\right)\right] .
\end{aligned}
$$

The definition of harmonic currents will therefore involve not only the harmonic velocity components, but harmonic density components as well. To illustrate the implication of this, let us evaluate the thirdharmonic component of the transverse current defined by

$$
j_{x 3}=-e\left(n_{0} v_{x 3}+n_{2} v_{x 1}\right)
$$

with $v_{x 1}$ and $v_{x 3}$ taken from the first two terms of (3.9) and $n_{2}$ taken from (3.12), all of them in the limit $\Delta=0$.

$$
\begin{aligned}
j_{x 3} \simeq \frac{e n_{0} v_{0}}{24} \epsilon^{2} \sin 3(\omega t-\beta z) & \\
& -\frac{e n_{0} v_{0}}{4} \epsilon^{2} \sin (\omega t-\beta z) \cos 2(\omega t-\beta z)
\end{aligned}
$$

$$
\begin{array}{rl}
\simeq-\frac{1}{12} \epsilon^{2}\left(e n_{0} v_{0}\right) \sin 3 & 3(\omega t-\beta z) \\
+ & \frac{1}{8} \epsilon^{2}\left(e n_{0} v_{0}\right) \sin (\omega t-\beta z) .
\end{array}
$$

The result dramatizes the difficulties encountered in nonlinear analysis of this type, even when pursued from the naive point of view we have adopted here. The products of harmonic components contain both sum and difference terms, the latter feeding corrections back into the lower order components. Fortunately, however, if these corrections are viewed in terms of a characteristic perturbation parameter, they will always be of higher order than the dominant contribution to a given harmonic and should therefore be of little practical significance.

The intrinsic electric-magnetic field interaction can therefore be expected to generate a harmonic spectrum of currents whose dominant terms are given by

$\overrightarrow{j_{0}} \simeq \frac{1}{2} \epsilon\left(e n_{0} v_{0}\right)\left[\cos \phi+\left(\Delta^{-1}-\Delta\right) \sin \phi\right] \hat{\mathrm{e}}_{z}$

$\overrightarrow{j_{1}} \simeq\left(e n_{0} v_{0}\right)[\sin (\omega t-\beta z)+\Delta \cos (\omega t-\beta z)] \hat{\mathrm{e}}_{x}$

$\overrightarrow{j_{2}} \simeq \frac{1}{4} \epsilon\left(e n_{0} v_{0}\right)\left[\cos 2\left(\omega t-\beta z-\frac{1}{2} \phi\right)\right.$

$$
\left.-\frac{3}{2} \Delta \sin 2\left(\omega t-\beta z-\frac{1}{2} \phi\right)\right] \hat{\mathrm{e}}_{z}
$$

$\overrightarrow{j_{3}} \simeq-\frac{1}{12} \epsilon^{2}\left(e n_{0} v_{0}\right)[\sin 3(\omega t-\beta z)+\mathrm{O}(\Delta)] \hat{\mathrm{e}}_{x}$

$\overrightarrow{j_{2 n}} \propto \epsilon^{2 n-1} \hat{\mathrm{e}}_{z}$

$\overrightarrow{j_{2 n+1}} \cong \epsilon^{2 n} \hat{\mathrm{e}}_{x}$.

The third harmonic has been simplified in accordance with (3.14) and the last two entries summarize the strength and polarization of the even and odd harmonics respectively. The anticipated longitudinal drift current shows up in $\overrightarrow{j_{0}}$, while $\overrightarrow{j_{1}}$ is just the linear polarization current in the field of the primary wave. As expected, the transverse current contains only odd harmonics, and these decrease by a factor $\epsilon^{2}$ from one term to the next. These transverse currents can 
generate odd harmonic waves traveling in the direction of the primary wave, provided that the dispersion at the primary frequency is so small that $\beta_{3}=3 \beta_{1}$ over an appreciable distance. On the other hand, it is easy to show from Maxwell's equations that there is no magnetic field associated with the longitudinal currents, so there will be no radiated harmonic fields of even order.

The situation is quite different in the presence of a steady magnetic field. If, for example, the direction of the steady field coincides with the magnetic field of the wave, a traveling space-charge wave of order $\epsilon$ is produced at the primary frequency $\omega$ which, in turn, provides a source for a second-harmonic transverse wave.

The procedure used to find $v_{x}$ and $v_{z}$ in this section resembles that used by Maiman [1957] for calculating harmonic currents produced by prescribed cavity fields. Moreover, some currents of this type were obtained by Chen [1962] for the case in which $\nu_{c}=0$. Chen's results are incorrect, however, containing a spurious factor $\left(1+\omega_{p}^{2} / \omega^{2}\right)$ which resulted, effectively, from the introduction of an internal polarization field in addition to the impressed field.

\section{Electron Temperature Effects}

There are many interesting and important phenomena associated with the dependence of the effective collision frequency of the electrons in a plasma upon the electron temperature $T_{e}$, but only a few authors have considered the role of this nonlinearity in the production of harmonic currents [Vilenskii, 1953; Rosen, 1961; Visvanathan, 1962]. There are actually several other ways in which electromagnetic field-induced electron temperature variations can produce harmonic currents in a plasma, but since the modulation of the collision frequency is probably the most important, we will consider it in greatest detail.

An approximate solution of (2.5) can be obtained by assuming that in this equation, at least, the collision frequency $\nu_{c}$ is constant. Under this condition, and with $\vec{v}$ replaced by its zeroth-order approximation (2.7), the solution has been shown by Ginzburg and Gurevich [1960] to take the form

$$
\begin{aligned}
T_{e}=T_{0}+T_{E}\left[1+\frac{\left(\delta \nu_{c}^{2}-2 \omega^{2}\right) \delta}{(2 \omega)^{2}+\delta^{2} \nu_{c}^{2}} \cos 2(\omega t-\beta z)\right. \\
\left.+\frac{\omega \nu_{c}(2+\delta) \delta}{(2 \omega)^{2}+\delta^{2} \nu_{c}^{2}} \sin 2(\omega t-\beta z)\right]
\end{aligned}
$$

where

$$
T_{E}=\frac{e^{2} E_{0}^{2}(z)}{3 k m \delta\left(\omega^{2}+\nu_{c}^{2}\right)} .
$$

If the temperature relaxation time $\tau=1 / \delta \nu_{c}$ is much larger than the period $2 \pi / \omega$ of the exciting wave, then $\delta\left(\nu_{c} / \omega\right)<<1$ and (4.1) simplifies to

$$
\frac{T_{e}}{T_{0}}=1+\frac{T_{E}}{T_{0}}\left[1-\frac{1}{2} \delta \cos 2(\omega t-\beta z)+\frac{1}{2} \delta \Delta \sin 2(\omega t-\beta z)\right] .
$$

Now, the definition of the effective collision frequency $\nu_{c}$ involves the electron velocity distribution function (hence some average energy parameter which we take here to be the electron kinetic temperature $T_{e}$ ) and the force law governing the scattering interaction between the electrons and the heavy particles. Accordingly, it is convenient to adopt a heuristic expression for $\nu_{c}$ of the form

$$
\nu_{c}\left(T_{e}\right)=\nu_{c o}\left(T_{e} / T_{0}\right)^{\sigma}
$$

where $\nu_{c o}$ is the equilibrium value $\nu_{c}\left(T_{0}\right)$.

The parameter $\sigma$ depends upon the nature of the scattering interaction. For example, $\sigma=1 / 2$ for rigidsphere collisions appropriate to low energy scattering by molecules, $\sigma=0$ for the induced polarization interaction usually occurring at high energies, and $\sigma=-3 / 2$ for coulomb scattering by ions. In general, a value of $\sigma$ for a particular molecule and $T_{e}$ can be estimated from empirical curves of collision probability versus electron energy. Many such curves are given by Brown [1959].

We now assume that $T_{E} / T_{0}>>1$, which allows us to ignore the first term on the right of (4.3) and write (4.4) in the form

$\nu_{c}\left(T_{e}\right) \simeq \nu_{c}^{(0)}\left\{1-\frac{1}{2} \delta[\cos 2(\omega t-\beta z)-\Delta \sin 2(\omega t-\beta z)]\right\}^{\sigma}$

where

$$
\nu_{c}^{(0)}=\nu_{c o}\left(T_{E} / T_{0}\right)^{\sigma} .
$$

Since $\delta<<1$, equation (4.5) can be expressed as a power series in $\delta$ :

$$
\begin{gathered}
\nu_{c}\left(T_{e}\right) \simeq \nu_{c}^{(0)}-\nu_{c}^{(0)} \frac{\sigma}{2} \delta[ \\
+\cos 2(\omega t-\beta z)-\Delta \sin 2(\omega t-\beta z)] \\
+\nu_{C}^{(0)} \frac{\sigma(\sigma-1)}{8}
\end{gathered}
$$$$
[\cos 2(\omega t-\beta z)-\Delta \sin 2(\omega t-\beta z)]^{2}+\ldots
$$

or

$$
\nu_{c}\left(T_{e}\right) \simeq \nu_{c}^{(0)}+\delta \nu_{c}^{(1)}+\delta^{2} \nu_{c}^{(2)}+\ldots .
$$

where the $\nu_{c}^{(n)}$ are identified by comparison with the coefficients of $\delta^{n}$ in (4.7).

Let us again take a reduced form of (2.3) in which $\vec{v} \cdot \nabla \vec{v}, \nabla p_{e}$, and now $\vec{v} \times \vec{B}$ are all ignored, and attempt a perturbation analysis based on an expansion of $\vec{v}$ in the form

$$
\vec{v}=\overrightarrow{v^{(0)}}+\overrightarrow{\delta v^{(1)}}+\vec{\delta}^{2 v^{(2)}}+\ldots .
$$


If we substitute (4.8) and (4.9) into

$$
\frac{\partial \vec{v}}{\partial t}=-\frac{e}{m} \vec{E}(z, t)-\nu_{c}\left(T_{e}\right) \vec{v}
$$

and equate successive powers of $\delta$, the coefficients in (4.9) are found to satisfy the equations

$$
\begin{aligned}
& \frac{\partial v^{(0)}}{\partial t}+\nu_{c}^{(0)} \overrightarrow{v^{(0)}}=-\frac{e}{m} \vec{E}(z, t) \\
& \overrightarrow{\partial v^{(1)}}+\nu_{c}^{(0)} v^{(1)}=-\nu_{c}^{(1)} \overrightarrow{v^{(0)}} \\
& \overrightarrow{\partial t}\left(v^{(2)}\right. \\
& \partial t
\end{aligned}
$$

The solution for the unperturbed velocity $\vec{v}^{(0)}$ is just (2.7) with $\hat{\kappa}=\hat{e}_{z}$ and $\nu_{c}$ replaced by $\nu_{c}^{(0)}$, while the solution for each higher-order coefficient requires knowledge only of the lower-order coefficients. Sacrificing generality for clarity, we write below the solutions for the first three coefficients in the special case $\sigma=\frac{1}{2}$ :

$$
\begin{array}{r}
\overrightarrow{v^{(0)}} \simeq-\overrightarrow{v_{0}}[\sin (\omega t-\beta z)+\Delta \cos (\omega t-\beta z)] \\
\overrightarrow{v^{(1)}} \simeq-\frac{1}{8} \overrightarrow{v_{0}} \Delta\left[\cos (\omega t-\beta z)-\frac{1}{3} \cos 3(\omega t-\beta z)\right] \\
\overrightarrow{v^{(2)}} \simeq+\frac{1}{64} \overrightarrow{v_{0} \Delta}\left[\cos (\omega t-\beta z)-\frac{1}{6} \cos 3(\omega t-\beta z)\right. \\
\left.+\frac{1}{10} \cos 5(\omega t-\beta z)\right] .
\end{array}
$$

Terms of order $\Delta^{2}$ are again ignored, and $\vec{v}_{0}=e \vec{E}_{0}(z) / m \omega$.

The velocity coefficients are all transverse, so there are no oscillating density components and the total current is therefor simply $\vec{j}=-e n_{0} \vec{v}$, which separates into the following harmonic components:

$$
\begin{gathered}
\overrightarrow{j_{c 1}} \simeq e n_{0} \overrightarrow{v_{0}}\left[\sin (\omega t-\beta z)+\Delta\left(1+\delta / 8-\delta^{2} / 64\right)\right. \\
\cos (\omega t-\beta z)] \\
\overrightarrow{j_{c 3}} \simeq-e n_{0} \overrightarrow{v_{0}}(\delta \Delta / 24)(1-\delta / 16) \cos 3(\omega t-\beta z) \\
\overrightarrow{j_{c 5}} \simeq e n_{0} \vec{v}_{0}\left(\delta^{2} \Delta / 640\right) \cos 5(\omega t-\beta z) \\
---- \\
\overrightarrow{j_{c(2 n+1)}} \propto \overrightarrow{v_{0}} \delta^{n} \Delta .
\end{gathered}
$$

Modulation of $\nu_{c}$ by the primary wave thus produces an odd harmonic spectrum of purely transverse currents whose magnitudes fall off by a factor $\delta$ from one to the next. An additional numerical factor enters from the series (4.7) and the subsequent integrations. This factor depends on the value of $\sigma$, and it turns out, for example, that in the case of collisions with ions $(\sigma=-3 / 2)$ the currents can be obtained from (4.15)(4.17) simply by replacing $\delta$ and $\delta^{2}$ with $-3 \delta$ and $-15 \delta^{2}$ respectively. Incidentally, the perturbation of order $\delta$ shown in the primary current in (4.15) was found by Taylor [1961] and by Epstein [1960] using a more complicated formulation of the problem.

Returning to (4.3), we see that a second-harmonic temperature wave accompanies the primary wave and ought to produce a longitudinal diffusion current through the $\nabla p_{e}$ term in (2.3). The appropriate velocity components can be estimated by neglecting $\vec{v} \cdot \nabla \vec{v}, v \times \vec{B}$ and $\nu_{c}\left(T_{e}\right) \vec{v}$ and substituting $\nabla p_{e}=k n_{0} \nabla T_{e}$ with $T_{e}$ given by (4.3).

The solution is given by

$$
\begin{array}{r}
v_{\mathrm{z}} \simeq \frac{2}{3} \frac{\epsilon}{\delta \Delta} v_{0} \tan \phi-\frac{1}{6} \epsilon v_{0}\left[\cos 2\left(\omega t-\beta \mathrm{z}-\frac{1}{2} \phi\right)\right. \\
\left.-\frac{3}{2} \Delta \sin 2\left(\omega t-\beta \mathrm{z}-\frac{1}{2} \phi\right)\right] .
\end{array}
$$

The second harmonic term is exactly of the same form as that found in the last section for the intrinsic interaction. Higher-order longitudinal harmonics could be found by reinstating the $\vec{v} \cdot \nabla \vec{v}$ term in (2.3) and proceeding with a perturbation calculation. There is no point in doing it here. It is sufficient to observe that such terms would, as before, generate even harmonics of order $\epsilon^{2}$ in the density $n$ as well as numerical corrections in the higher-order components of $\vec{v}$, but would not be expected to alter the general features of the transverse currents in any important way. The zeroth-order term in (4.19) is a diffusion velocity resulting from the decay of the field amplitude (hence $T_{e}$ ) as the wave moves through the slightly lossy plasma. Although its existence is of interest, its form in (4.19) cannot be taken seriously. If the electrons are urged to flow in the direction of wave propagation by a temperature gradient, the resulting charge separation introduces space-charge forces and the problem becomes one of ambipolar diffusion.

Finally, we should mention the effect of temperature oscillations on such chemical rate processes as ionization, recombination, and attachment. These processes can be included by introducing energy dependent coefficients in rate terms added to the right side of (2.4):

$\frac{\partial n}{\partial t}+\nabla \cdot(n \vec{v})=\nu_{i}\left(T_{e}\right) n-\alpha_{r}\left(T_{e}\right) n^{2}-\nu_{a}\left(T_{e}\right) n$.

The rate coefficients are the ionization frequency $\nu_{i}$, the recombination coefficient $\alpha_{r}$ and the attachment frequency $\nu_{a}$. The perturbation procedure used earlier to find the effects due to $\nu_{c}\left(T_{e}\right)$ can be applied to $(4.20)$ as well, and the results are much the same. As an example, we have calculated the dominant thirdharmonic current due to the temperature dependence of the recombination coefficient $\alpha_{r}$. The result is

$$
\overrightarrow{j_{r 3}}=C\left(e \mathbf{n}_{0} \overrightarrow{v_{0}}\right) \delta\left(\nu_{r o} / \omega\right) \cos 3(\omega t-\beta z)
$$


where $C$ is a numerical constant and $\nu_{r 0}$ is the zerothorder recombination frequency defined by $\nu_{r 0}$ $=\alpha_{r}\left(T_{E}\right) n_{0}$. The other coefficients in (4.20) would contribute harmonic currents of similar form.

The experimental results obtained by Baird and Coleman [1961] remain a puzzle. They found both odd and even harmonics radiated from a microwave discharge between two posts in a waveguide when an additional d-c electric bias field was applied. Their measurements were remarkably consistent with the predictions of a simple theory based on the assumption that $\nu_{i}=\gamma|\vec{v}|$ on the right side of $(4.20)$, where $\gamma$ is a constant and $\vec{v}$ is simply the zeroth-order electron velocity in the combined a-c and d-c electric fields. Unfortunately, there is no convincing justification for this assumption, (their simple argument using a onedimensional velocity distribution function contains an error). Our formulation would also lead to both odd and even harmonics in combined a-c and d-c electric fields, but in order to obtain anything resembling the relation $\nu_{i} \sim|\vec{v}|$, the condition on the solution of $(2.5)$ would have to be $\delta \nu_{c} \gg \omega$, which is difficult to satisfy at microwave frequencies.

\section{Effect of Plasma Inhomogeneity}

Up until now, our discussion of nonlinear interactions has supposed that the plasma was homogeneousat least to first order. If the plasma is inhomogeneous to start with, it becomes impossible to prescribe the primary wave without considering a specific case for which a solution, generally of a complicated propagation problem, is available. However, the essential feature of an inhomogeneous plasma is its nonvanishing density gradient, so we can get some idea of the character of harmonic currents in plasma inhomogeneities by studying what happens when a quasi-plane wave encounters a region in which $\nabla n_{0} \neq 0$.

In order to maintain a connection with our previous discussion, we suppose that the wave propagating in the inhomogeneous plasma may be written in the form

$$
\vec{E}(\vec{r}, t)=\overrightarrow{E_{0}}(\overrightarrow{\mathrm{r}}) \cos (\omega t-\vec{k}(\vec{r}) \cdot \vec{r})
$$

where $\vec{k}(r)$ is the wave vector. Such an expression for $\vec{E}$ is generally valid only in the limit of geometrical optics. The average velocity of the electrons under the influence of this field will be taken to be the zerothorder approximation (2.7) in the limit of small $\Delta$. To avoid confusion with $\vec{v}^{(0)}$ in previous sections, we will write this as

$$
\begin{aligned}
\vec{v}^{\prime} \simeq-\frac{e}{m \omega} \vec{E}_{0}(r)[\sin (\omega t-\vec{k} \cdot \vec{r}) & \\
& +\Delta \cos (\omega t-\vec{k} \cdot \vec{r})] .
\end{aligned}
$$

Since $\nabla n_{0} \neq 0$ we will need (2.4) at the outset, which requires that

$$
\frac{\partial n}{\partial t}+\nabla n \cdot \vec{v}^{\prime}+n \nabla \cdot v^{\prime}=0 .
$$

In general, the second and third terms in (5.3) will not vanish and there must therefore be a time-varying component of electron density. Considering this component to be a perturbation on the original electron density $n_{0}(\vec{r})$ of the inhomogeneous plasma, we write

$$
n=n_{0}(\vec{r})+n^{(1)}(\vec{r}, t)
$$

and substitute into (5.3), yielding

$$
\frac{\partial n^{(1)}}{\partial t}+\nabla n_{0} \cdot \vec{v}^{\prime}+n_{0} \nabla \cdot \vec{v}^{\prime}=0
$$

as the equation for $n^{(1)}$. Since it has been assumed that the original electron distribution $n_{0}(\vec{r})$ is perfectly neutralized by a corresponding ion distribution and, moreover, that the ions remain unmoved by the field, then $n^{(1)}$ must represent an oscillating space charge. It is easy to see how this space charge comes about. Let us count electrons in a small volume located within the inhomogeneity, keeping in mind that the ion population in this volume will remain stationary. If the primary field forces electrons to move down the density gradient, the volume will acquire a net negative charge as electrons arrive in it from regions of higher density. Conversely, if the electrons are forced up the gradient the electron population in the little volume comes from regions of lower density, and since the ions remain fixed, the net charge is positive. For a wave-like driving field there will be a wavelike space charge perturbation moving along the inhomogeneity.

The existence of space charge implies the existence of a longitudinal component of the electric field, that is $\vec{E}$ must satisfy

$$
\nabla \cdot \vec{E}(\vec{r}, t)=-e n^{(1)} / \epsilon_{0}
$$

Since it is assumed that (5.1) is a proper solution of the propagation problem, we may use (5.6) and (5.2) to deduce that

$$
\nabla \cdot \vec{v}^{\prime}=\frac{e^{2}}{\epsilon_{0} m \omega^{2}}\left[\nu_{c} n^{(1)}-\frac{\partial n^{(1)}}{\partial t}\right]
$$

which, when substituted into (5.5), yields

$$
\left(1-\frac{\omega_{p}^{2}}{\omega^{2}}\right) \frac{\partial n^{(1)}}{\partial t}+\left(\frac{\omega_{p}^{2}}{\omega^{2}}\right) \nu_{c} n^{(1)}=-\nabla n_{0} \cdot \vec{v}^{\prime}
$$

where we have introduced the plasma frequency $\omega_{p}^{2}=e^{2} n_{0} / \epsilon_{o} m$. The steady-state solution of (5.8) when $\omega_{p}<\omega$ is found to be

$$
\begin{aligned}
n^{(1)}= & \frac{(e / m) \nabla n_{0} \cdot \overrightarrow{E_{0}}}{\left(\omega^{2}-\omega_{p}^{2}\right)^{2}+\left(\omega_{p}^{2} \Delta\right)^{2}}\left\{\left[\left(\omega^{2}-\omega_{p}^{2}\right)-\omega_{p}^{2} \Delta^{2}\right]\right. \\
& \left.\cos (\omega t-\vec{k} \cdot \vec{r})-\omega^{2} \Delta \sin (\omega t-\vec{k} \cdot \vec{r})\right\}
\end{aligned}
$$

or, if terms of order $\Delta^{2}$ are ignored and the abbreviation $\epsilon=\left(1-\omega_{p}^{2} / \omega^{2}\right)$ is used, 


$$
\begin{aligned}
n^{(1)} \simeq \frac{e \nabla n_{0} \cdot \overrightarrow{E_{0}}}{m \omega^{2} \epsilon}[\cos (\omega t & -\vec{k} \cdot \vec{r}) \\
& \left.-\frac{\Delta}{\epsilon} \sin (\omega t-\vec{k} \cdot \vec{r})\right] .
\end{aligned}
$$

It should be remembered that $n_{0}, \overrightarrow{E_{0}}, \epsilon$ and $\vec{k}$ are all functions of position $\vec{r}$.

A nonlinear current is produced when these space charges interact with the field. Using (5.2) and (5.10) in $\vec{J}^{(1)}=-e n^{(1)} \vec{v}^{\prime}$, we get

$$
\begin{aligned}
& \overrightarrow{j^{(1)}} \simeq \frac{1}{2} \Delta \frac{\omega_{p}^{2}}{\omega^{2}} \frac{e^{3} \nabla n_{0} \cdot \overrightarrow{E_{0}}}{m^{2} \omega^{3} \epsilon^{2}} \vec{E}_{0}(\vec{r})-\frac{1}{e^{3} \nabla n_{0} \cdot \vec{E}_{0}} \vec{E}_{0}(\vec{r}) \\
& {\left[\sin 2(\omega t-\vec{k} \cdot \vec{r})+\frac{(1+\epsilon)}{\epsilon} \Delta \cos 2(\omega t-\vec{k} \cdot \vec{r})\right] \text {. }}
\end{aligned}
$$

The first term is a d-c drift current in the direction of the field vector $\overrightarrow{E_{0}}$; the second term is a second harmonic current, also in the direction of $\overrightarrow{E_{0}}$. Notice that if the inhomogeneous region of the plasma contains a point of plasma resonance $\left(\omega_{p}=\omega\right)$, the current increases sharply in the neighborhood of this point due to the vanishing of $\epsilon$ in the denominator. Under these conditions one must return to the more complete expression for $n^{(1)}$ given in (5.9). The second harmonic current in (5.11) is then large but bounded by collisions when $\omega_{p}=\omega$, having the expression

$$
\begin{aligned}
\overrightarrow{j_{2}^{(1)}} \simeq-\frac{1}{2} \frac{e^{3} \nabla n_{0} \cdot \overrightarrow{E_{0}}}{m^{2} \omega^{3} \Delta} \vec{E}_{0}(\vec{r})[\cos 2(\omega t-\vec{k} \cdot \vec{r}) \\
-2 \Delta \sin 2(\omega t-\vec{k} \cdot \vec{r})] .
\end{aligned}
$$

In order to have any confidence in the evaluation of this type of nonlinear interaction in inhomogeneous plasmas for which $\omega_{p} \sim \omega$, it is really necessary to solve (2.3) to (2.5), or some equivalent system, along with Maxwell's equations for the field. In the vicinity of plasma resonance, for example, the field amplitude $\overrightarrow{E_{0}}(r)$ will itself be strongly inhomogeneous, and therefore the electrons will be subjected to a force of changing magnitude along their trajectories. This leads to strongly anharmonic oscillations over a narrow region and could thereby produce harmonic waves [Försterling and Wüster, 1951]. The sinusoidal motion of an electron will be distorted only to the extent that its displacement from equilibrium is large enough for the electrons to "see" the field inhomogeneity.

If we indroduce a length $\Lambda_{E}$ to measure the distance over which the amplitude of the electric field changes appreciably, and use $\langle\xi\rangle$ to denote the maximum displacement of an electron in the average field in the neighborhood of a point, then the ratio $\langle\xi\rangle / \Lambda_{E}$ is a measure of the importance of this nonlinearity. Field inhomogeneity has occasionally been proposed as a source of harmonics in microwave discharges [Swan, 1961 and references cited there], but to consider it further would carry us beyond the scope of our present discussion.
If the plasma is underdense, in the sense that $\omega_{p}^{2}$ is always much smaller than $\omega^{2}$, then $\epsilon \simeq 1$ and the amplitude of the primary wave varies slowly with position. This means that $(5.2)$ can be integrated locally to give the electron displacement $\vec{\xi}=\int \vec{v}^{\prime} d t$ at $\vec{r}$ When $\Delta=0$, the magnitude of this displacement is just

$$
\xi=\frac{e E_{0}}{m \omega^{2}}
$$

Considering the case of strongest interaction in which $\overrightarrow{E_{0}}$ is parallel to $\nabla n_{0}$, and estimating the local nonuniformity of the plasma by a characteristic length $\Lambda_{n}$ defined by $\left|\nabla n_{0}\right|=n_{0} / \Lambda_{n}$, the magnitude of the second harmonic current in (5.11) may be written as

$$
\begin{aligned}
\left|j_{2}^{(1)}\right| \simeq e n_{0}\left[\frac{e E_{0}}{m \omega}\right) & {\left[\frac{e E_{0}}{2 m \omega^{2} \Lambda_{n}}\right] } \\
& \left.\simeq \mid e n_{0} v^{\prime}\right)\left(\xi / 2 \Lambda_{n}\right)=\left|j_{1}\right|\left(\xi / 2 \Lambda_{n}\right)
\end{aligned}
$$

where $\left|j_{1}\right|$ is the magnitude of the primary current. Clearly, the perturbation parameter that emerges here is $\left(\xi / 2 \Lambda_{n}\right)$, which is the ratio of the maximum electron displacement in the field to the scale of the density gradient. This parameter is usually quite small.

As pointed out elsewhere by one of us [Wetzel, 1963], the dominant harmonics produced by this interaction form a sequence in which the $p$ th-harmonic current is $\overrightarrow{j_{p}}=-e n_{p-1} \vec{v}^{\prime}$, where the electron density component $n_{p-1}$ is obtained from

$$
\frac{\partial n_{p-1}}{\partial t}+\nabla \cdot \overrightarrow{j_{p-1}^{(1)}}=0
$$

Under special conditions this sequence contains both odd and even harmonics which fall off from one term to the next by the factor $\left(\xi / 2 \Lambda_{n}\right)$. It is clear, however, that the spectrum is determined by the way in which $n_{0}(\vec{r})$ changes with position along the direction of the primary electric field vector, so it is impossible to make any general statements about the relative amplitudes of the harmonic components. Various aspects of this type of nonlinear interaction in an inhomogeneous plasma, including the effect of a steady magnetic field on $n^{(1)}$, may be found in papers by Ginsburg [1958] and by Wetzel [1961a, b, 1963].

There is still another nonlinear current which has nothing to do with space charge, but is associated with inhomogeneities and properly belongs among the electron-temperature effects discussed in the last section. In an inhomogeneous plasma, the pressure gradient in (2.3) consists of two terms

$$
\nabla\left(n_{0} k T_{e}\right)=n_{0} k \nabla T_{e}+k T_{e} \nabla n_{0} .
$$

The first term was treated in section 4 , but the second term gives rise to another second harmonic of electron velocity which, when multiplied by $-e n_{0}$, provides a 
current

$$
\begin{aligned}
\overrightarrow{j_{2}} \simeq-\frac{1}{12} \frac{e^{3} E_{0}^{2}}{m^{2} \omega^{3}} \nabla n_{0}[ & \sin 2(\omega t-\vec{k} \cdot \vec{r}) \\
& \left.+\frac{3}{2} \Delta \cos 2(\omega t-\vec{k} \cdot \vec{r})\right] .
\end{aligned}
$$

This current is the same size as the corresponding space charge current, but is directed along the density gradient rather than along the electric field vector. Its origin in (5.16) shows that it is a harmonic component of the diffusion current due to concentration gradients and should therefore be regarded with suspicion, in view of the discussion following (4.19) concerning diffusion currents.

\section{Summary}

This review of the major sources of induced harmonic currents in an isotropic plasma has shown that these sources are quite weak and that the harmonic currents are all measured by small perturbation parameters of some kind. We summarize the main results of the preceding sections below.

A. Intrinsic $\vec{E}-\vec{B}$ interaction: Odd harmonic transverse currents of relative magnitudes $\left|j_{2 n+1}\right| \propto \epsilon^{2 n}\left|j_{1}\right|$, where $\epsilon=\left(v_{0} / c_{p}\right)$ is the ratio of the unperturbed electron velocity in the field of the wave to the phase velocity of the wave; even harmonic nonradiating longitudinal currents plus a drift current. The parameter $\epsilon$ can be estimated from the formula $\epsilon \simeq 10^{-4}$ $\left(E_{0} / f\right)$, where $E_{0}$ is given in volts/meter and $f$ is given in megacycles.

B. Electron temperature effects: Odd harmonic transverse currents of relative magnitudes $\left|j_{2 n+1}\right|$ $\propto \delta^{n} \Delta\left|j_{1}\right|$, where $\delta$ is the energy loss parameter and $\Delta$ is the ratio $\left(\nu_{c} / \omega\right)$; even harmonic longitudinal currents resembling those under $A$. above. It should be noted that the results of section 4 are based on the assumption of a field strong enough to raise the electron temperature well above that of the heavy particles, but that this is usually accomplished even at fairly weak field strengths [Ginsburg and Gurevich, 1960]. Under these conditions the spectral amplitudes do not depend explicitly on the strength of the field. For elastic collisions, $\delta=2 \mathrm{~m} / \boldsymbol{M}$ which is or order $10^{-5}$, but in an excited gas $\delta$ can become much larger, even approaching $10^{-1}$ in some cases (see the reference cited above).

C. Space-charge effects in plasma inhomogeneities: Possible quasi-transverse currents at all harmonics. The relative amplitudes depend upon both the functional dependence of $n_{0}$ on $\vec{r}$ and the direction of the primary electric field, but are related to the parameter $\left(\xi_{M} / \Lambda_{n}\right)$, where $\xi_{M}$ is the maximum displacement of an electron in the field and $\Lambda_{n}$ is a length measuring the distance over which the electron density changes appreciably. These currents can be expected to be most prominent in regions of rapidly changing plasma density and in the neighborhood of a point of plasma resonance.

\section{References}

Armstrong, J. A., N. Bloembergen, J. Ducuing, and P. S. Pershan (1962), Interaction between light waves in a nonlinear dielectric, Phys. Rev. 127, 1918-1940.

Baird, J. R., and P. D. Coleman (1961), Frequency conversion in a microwave discharge, Proc. IRE 49, No. 12, 1890-1900.

Bloembergen, N. (1963), Wave propagation in nonlinear electromagnetic media, Proc IEEE 51, No. 1, 124-131.

Brown, S. C. (1959), Basic Data of Plasma Physics Chap. 1 (John Wiley and Sons, New York, N.Y.).

Chen, K. M. (1962), Interaction of a high-intensity EM field with a low-density plasma, IRE Trans. AP-10, No. 1, 31-42.

Epstein, M. (1960), Nonlinear behavior of the electrical conductivity of a slightly ionized gas, Phys. Fluids 3, No. 6, 1016-1018.

Fain, V. M. (1955), The velocity distribution of electrons in the presence of a varying electric field and a constant magnetic field, J. Exper. Theoret. Phys. USSR 28, 422-430.

Feinstein, J. (1950), Higher order approximations in ionospheric propagation, J. Geophys. Res. 55, 161-170.

Försterling, K., and H. Wüster (1951), Uber die Entstehung von Oberwellen in der Ionospäre, J. Atmospheric Terrest. Phys. 2, $22-31$.

Ginsburg, V. L. (1958), Nonlinear interaction of radio waves propa gating in a plasma, J. Exper. Theoret. Phys. USSR 35, 1573-1574.

Ginsburg, V. L., and A. V. Gurevich (1960), Nonlinear phenomena in a plasma located in an alternating electromagnetic field, Usp. Fiz. Nauk 70, 201-246 and 393-428.

Maiman, T. H. (1957), Solid State Millimeter Wave Generation Study, Hughes Aircraft Company Physics Laboratory, Final Report on Contract DA 36-039SC-73063.

Margenau, H., and L. M. Hartman (1948), Theory of high-frequency discharges. II. Harmonic components of the distribution function, Phys. Rev. 73, No. 4, 309-315.

Rosen, P. (1961), Generation of the third harmonic by an electromagnetic signal in a plasma, Phys. Fluids 4, No. 3, 341-344.

Rydbeck, O. E. H. (1963), Dynamic nonlinear electromagnetic wave propagation and harmonic radiation in magnetoionic media, Conference on Wave Interaction and Dynamic Nonlinear Phenomena in Plasmas, Pennsylvania State University Engineering Proceedings $\mathrm{P}-42,42-70$.

Swan, C. B. (1961), Generation of microwave harmonics in and electrodeless discharge at low pressure, Proc. IRE 49, No. 12, 19411942.

Taylor, L. S. (196i), Conductivity of slightly ionized gases, Phys. Fluids 4, No. 12, 1499-1503.

Uenohara, M., T. Masutani, and K. Inada (1957), A new high-power frequency multiplier, Proc. IRE 45, 1419-1420.

Vilenskii, I. M. (1953), The effect of nonlinearities on radio waves propagating in the ionosphere, Doklady Akad. Nauk USSR 92, $525-528$.

Visvanathan, S. (1962), Electron temperature in partially ionized gases subject to intense ac fields, J. Appl. Phys. 33, 2481-2483.

Wetzel, L. (196la), Wave interaction in plasma inhomogeneities, J. Appl. Phys. 32, No. 2, 327-328.

Wetzel, L. (1961b), Electric-field-induced anisotropies in an inhomogeneous plasma, Phys. Rev. 123, No. 3, 722-726.

Wetzel, L. (1963), Harmonic excitation in and reradiation from nonuniform ionized regions, Symposium on Electromagnetic Theory and Antennas, ed. E. C. Jordan, (Pergamon Press, Oxford), 365-374.

Whitmer, R. F., and E. B. Barret (1961), Nonlinear interaction of an electromagnetic wave with a plasma layer in the presence of a static magnetic field. I. Theory of harmonic generation, Phys. Rev. 121, 661-668. 